\title{
The Linear Factorial Smoothing for the Analysis of Incomplete Data
}

\author{
Basavanneppa Tallur \\ IRISA, Université de Rennes 1, Avenue Général Leclerc, \\ 35042 Rennes Cedex, France \\ tallur@irisa.fr \\ http://www.irisa.fr/symbiose/people/tallur
}

\begin{abstract}
Huge amounts of data are generated in every field of science and technology and the need for the proper data analysis tools and their adaptation to the ever-increasing data size is more and more crucial. Statistical exploratary data analysis techniques -such as principal component analysis, correspondence analysis, clustering and classification among others- are greatly useful in discovering useful information -or knowledge- hidden in data but they require the data set to be complete. In many situations the data is incomplete for various reasons. Erroneous and uncertain data may also be considered as missing since their use may lead to incorrect results. Many research works have addressed this issue in specific applications. This paper presents a simple and efficient iterative method for estimating the missing values in the data set based on linear factorial smoothing. Though this work was prompted by the recurrent problem faced in the field of bioinformatics while analysing the gene expression data, the method proposed for missing value imputation in this paper may be useful in any area.
\end{abstract}

\section{Introduction}

Statistical data analysis techniques are gaining more and more importance with the tremendous development in information technology. These techniques are also getting more and more computation-intensive. Most data need to be preprocessed before any analysis is carried out so as to obtain reliable results. One of the recurrent problems in all areas of data analysis applications is that of missing values. The reasons are varied and multiple, such as : non-response to a questionnaire, failure of a measuring apparatus, physical impossibility to observe a value, just to enumerate a few. Erroneous measurements and doubtful observations may also be treated as missing data since such data may lead to incorrect conclusions. We consider the widely used multidimensional data analysis techniques which are very fundamantal in exploring huge data sets in order to discover useful information ; they are part of what is called the data mining engine. Many researchers in different areas of application of statistical data analysis techniques have proposed different strategies for dealing with the missing values before performing the analyses. These strategies range from simply 
deleting the entire observations (i.e. rows in a data table) containing missing values, to estimating the missing values by some elaborate optimization techniques. We consider that the method of missing value imputation should take into consideration the type of analysis that will follow. The idea is that if one needs to perform a supervised classification, then one has to estimate the missing values in such a way that the classifiers resulting from the estimated data be as close as possible to the real classifiers i.e. the classifiers obtained from the complete data. Similarly, if one intends to apply principal component analysis (PCA), the estimated data should produce the principal factors as similar as possible to the factors the complete data would have produced. In this paper, we will describe an iterative smoothing method to estimate the missing values for PCA and correspondence analysis (CA) which are the basic techniques used in dimension reduction prior to other analyses such as clustering and classification. In order to assess the quality of the estimation we have used the complete data sets in which some of the randomly chosen values are considered as missing. The results obtained from both known complete data set and the estimated one are compared.

One particular area of research in which the data analysis tools are indispensable is bioinformatics where the data is generated in very large scales, and typically, these data sets contain missing values. In postgenomic research, gene expression microarray experiments can generate data sets with many missing values. Clustering techniques are widely used in gene expression data analysis because, by clustering the genes based on the similarity of their expression profiles the biologists may find functionally related genes and potentially the function of the genes (cf. Eisen et al. [3], Mao et al. 6], Tallur [11]). The recent research in bioinformatics shows that the linear factorial model allows interpretation in terms of biological processes (see e.g. Alter et.al. [1, Girolami and Breitling [2], Lee and Batzoglou [4], Roberts and Everson [7]). To address the problem arising from the high dimensional data, S. Roweis [8] has proposed the EM algorithms. These algorithms naturally accomodate missing data. A more recent survey on missing data estimation methods as applied in bioinformatics is given by Troyanskaya et al. [12. In the next sections we will briefly describe the principles of PCA and CA before presenting the algorithm of missing value estimation. Finally, we will present the experiments on which the algorithm has been tested.

\section{Principal Component Analysis (PCA)}

Let us consider a rectangular data matrix $X$ having $n$ rows (each row corresponds to one observation or data point) and $p$ columns (each column corresponds to a variable or an attribute). Let $X_{1}, X_{2}, \ldots, X_{p}$ be the variables. Principal component analysis is the fundamental technique for dimension reduction based on the principle of singular value decomposition of the data matrix. This analysis is appropriate when variables are numerical or quantitaive. In order to obtain the statistically interpretable results, the data matrix is standardized with respect 
to the mean and standard deviation of each variable. Moreover, the euclidean distance between data points is independent of measurement units when the variables are standardized.

\subsection{Formulation of PCA}

Each data point is represented as a vector in a $p$-dimensional vector space. The principal factorial axes are defined by the normalized eigenvectors of the $p \times p$ correlation matrix $R=\left(\left(r_{j k}\right)\right)_{j, k=1, \ldots, p}$ where $r_{j k}$ is the correlation coefficient between $X_{j}$ and $X_{k}$.

\subsection{Data Reconstruction from Principal Factors}

From the normed eigenvectors $u_{j},(j=1, \ldots, k)$, relative to the $k$ largest eigenvalues $\lambda_{j},(j=1, \ldots, k)$ of the matrix $R$ and the first $k$ principal components $c_{j}$, for $j=1, \ldots, k$, (with $k<p$ ) the data matrix $X$ may be reconstructed approximately as follows :

$$
X=\sum_{j=1}^{k} c_{j} u{ }_{j}
$$

where $u{ }_{j}$ is the transpose of the vector $u_{j}$. In other words, the estimated value of $X_{j}$ for $i^{\text {th }}$ observation is given by

$$
x_{i j}=\sum_{l=1}^{k} c_{l i} u_{l j} \text { for } i=1, \ldots, n \text { and } j=1, \ldots, p .
$$

We will call the integer $k$ the level of approximation.

\section{Correspondence Analysis (CA)}

The CA may be considered as an adaptation of PCA applicable to the contingency tables; the rows and columns of the data table correspond to the values (called modalities) of two qualitative variables, say, $Q_{1}$ and $Q_{2}$; and the $(i, j)^{t h}$ cell contains the frequency $x_{i j}$ (i.e. the number of simultaneous occurrences) of the $i^{\text {th }}$ modailty of $Q_{1}$ and the $j^{\text {th }}$ modailty of $Q_{2}$. Though the CA was developed originally in the framework of the contingency tables, it is widely applied to any data table with all positive values .

\subsection{Formulation of CA}

Before applying the PCA, the data is transformed so as to represent the rows and columns by their profile vectors. The $i^{\text {th }}$ row profile is a vector $\left(\frac{x_{i 1}}{x_{i}}, \ldots, \frac{x_{i p}}{x_{i .}}\right)$, in the $p$-dimensional vector space, where $x_{i}$. $=\sum_{j=1}^{p} x_{i j}$. The $i^{\text {th }}$ row profile is given the weight $p_{i .}=\frac{x_{i}}{x_{.}}$where $x_{. .}=\sum_{i=1}^{n} \sum_{j=1}^{p} x_{i j}$. Analogously, the $j^{\text {th }}$ column profile is a vector $\left(\frac{x_{1 j}}{x_{. j}}, \ldots, \frac{x_{n j}}{x_{. j}}\right)$, in the $n$-dimensional vector space 
where $x_{. j}=\sum_{i=1}^{n} x_{i j}$. The $j^{t h}$ column profile is given the weight $p_{. j}=\frac{x_{. j}}{x_{. .}}$. The $\chi$-squared metric is used to measure the distance btween row profiles in this row vector space ; according to this metric, the distance between the row profiles $i$ and $i$ is given by :

$$
d^{2}(i, i \prime)=\sum_{j=1}^{p} \frac{1}{p_{. j}}\left(\frac{x_{i j}}{x_{i .}}-\frac{x_{i \prime j}}{x_{i \prime .}}\right)^{2}
$$

Similarly, the distance between the column profiles $j$ and $k$ is :

$$
d^{2}(j, k)=\sum_{i=1}^{n} \frac{1}{p_{i .}}\left(\frac{x_{i j}}{x_{. j}}-\frac{x_{i k}}{x_{. k}}\right)^{2}
$$

With this set up which is perfectly symmetrical with respect to rows and columns, the PCA is applied to the row and column spaces. The row factors $F_{\alpha},(\alpha=$ $1,2, \ldots, p-1)$ are defined by the normed eigenvectors of the matrix $S$ whose general term is $s_{j k}$ :

$$
S=\left(\left(s_{j k}\right)\right)_{j, k=1, \ldots, p} \text { where } s_{j k}=\sum_{i=1}^{n}\left(\frac{x_{i j} x_{i k}}{x_{i .} x_{. k}}\right)-p_{. j}
$$

By interchanging the rows and columns, one can obtain the column factors $G_{\alpha}$. For further details the readers may be referred to the book by Lebart et.al. [5]

\subsection{Data Reconstruction from Factors}

The original data matrix may be reconstructed from the row and column factors to the order of approximation (or, appoximation level) $k$, with $k<p-1$ as follows :

$$
x_{i j}=\frac{x_{i .} x_{. j}}{x_{. .}}\left[1+\sum_{\alpha=1}^{k} \lambda_{\alpha}^{1 / 2} F_{\alpha}(i) G_{\alpha}(j)\right] \text { for } i=1, \ldots, n \text { and } j=1, \ldots, p .
$$

\section{Missing Value Estimation Algorithm}

There are two versions of the algorithm namely, ESTIMPCA and ESTIMCA depending on the ultimate analysis performed on the data. The former is appropriate for PCA and the latter for CA.

\subsection{ESTIMPCA Algorithm}

1. Initialization: Assign zeros (or the variable means, or medians) to each of the missing values and assign the value $s_{\max }$ to the parameter $s$, the approximation level. Note that $s_{\max }$ should be strictly less than $p$.

2. repeat the following steps until convergence :

(a) Compute the matrix $R$, and its eigenvalues. (Let $\lambda_{j},\left(j=1, \ldots, s_{\max }\right)$ be the $s_{\max }$ largest eigenvalues of $R$ ) ;

(b) Compute the corresponding eigenvectors $u_{j}$ and principal components $c_{j},\left(j=1, \ldots, s_{\max }\right)$.

(c) estimate each missing value $x_{i j}$ from equation (2) with $k=s_{\max }$ ). 


\subsection{ESTIMCA Algorithm}

1. Initialization: Assign zeros to each of the missing values and assign the value $s_{\max }$ to the parameter $s$, the approximation level. Note that $s_{\max }$ should be strictly less than $p-1$.

2. repeat the following steps until convergence :

(a) Compute the matrix $S$, and its eigenvalues. (Let $\lambda_{\alpha},\left(\alpha=1, \ldots, s_{\max }\right)$ be the $s_{\max }$ largest eigenvalues of $S$ );

(b) compute the row factors $F_{\alpha}$ and column factors $G_{\alpha},\left(\alpha=1, \ldots, s_{\max }\right)$.

(c) estimate the missing value $x_{i j}$ from equation (6) with $k=s_{\max }$.

\section{Experimental Validation}

In order to validate the alhoritms ESTIMPCA and ESTIMCA, we have used two real world data sets : the first one was obtained by the microarray experimentation conducted at the INSERM laboratory, Rennes (France) and the second one contains the data about the french agriculture. The former data set is particularly suitable for PCA and the latter for CA. Both data sets were complete.

\subsection{Gene Expression Data}

The first test data set contains gene expression levels of 2175 genes (rows) for each of 12 patients (columns). We generated random sample of upto 5220 numbers without replacement (that corresponds to $20 \%$ of the total number of data values) lying between 1 and 26,100. Then the values corresponding to the randomly chosen cells were considered as missing. The algorithm ESTIMACP with $s_{\max }=5$ produced not only the estimation of the missing values but also the estimated principal axes and principal components. Real components and the estimated ones were found to be very close to each other as measured by the high positive linear correlation coefficients, generally higher than 0.95 .

\subsection{Agricultural Data}

This data set contains the number of agricultural farms in each of the 89 french departmants (rows) having 8 different sizes (columns) ranging from the smallest (less than 1 hectare) to the largest (more than 100 hectares). The same method was used to simulate the missing values in the data table and the ESTIMCA algorithm was used for estimation. Again, upto $20 \%$ of the total 712 data values were randomly deleted before estimating them. We rather are interested in estimating the factors than the data values themselves. The results were found to be almost comparable to those of the first experiment.

\section{Conclusion and Remarks}

We have proposed a simple but efficient itertive method for estimating the missing values in view of a specific analysis - $\mathrm{PCA}$ and $\mathrm{CA}-$ which are generally 
considered as dimension reduction techniques prior to classification and/or clustering. This work was triggered by the real necessity that arose in gene expression data analysis. Moreover, it has been shown by the recent research works in bioinformatics that the linear factorial methods provide the biologically interpretable models. Each of the algorithms we have proposed necessitates the determination of the parameter $s_{\max }$ i.e., the number of factrors used for estimation. Higher the value of $s_{\max }$, faster the algorith will converge. But it should not be too close to $p$. Neither should it be too small a number. We may use a pilot study to determine this parameter.

\section{References}

1. Alter, O., Brown, P., Botstein, D.: Singular value decomposition for genome-wide expression data processing and modelling. PNAS 97 (2000) 10101-10106

2. Girolami, M., Breitling, R.: Biologically valid linear factor models of gene expression. Bioinformatics 20 (2004) 3021-3033

3. Eisen, M., Spellman, P., Brown, P., Botstein, D.: Cluster analysis and display of genome-wide expression patterns. PNAS. 95 (1998) 14863-14868

4. Lee, S.I., Batzoglou, S. Application of independent component analysis to microarrays. Genome Biol. 4 (2003)

5. Lebart, L., Morineau, A., Warwick, KM.: Multivariate descriptive statistical analysis, Correspondence analysis and related techniques for large matrices. Wiley series in probability and mathematical statistics (1984)

6. Mao, R., Zielke, C.L., Zielke, H.R., Pevnser, J. Global upregulation of chromosome 21 gene expression in the developing Down syndrome brain. Genomics 81 (2003) 457-467

7. Roberts, S., Everson, R. (eds) Independent component analysis Principles and practice. Cambridge university press, Cambridge (2001)

8. Roweis, S. EM Algorithms for PCA and SPCA. in Advances in neural informartion processing systems, 10 (1998)

9. Tallur, B. Analyse des correspondances en cas de données manquantes: application en biologie. Thèse doctorat de 3ème cycle, (1973) Université de Paris 6

10. Tallur, B. Contribution à l'analyse exploratoire de tableaux de contingence par la classification. Thèse doctorat ès science, (1988) Université de Rennes 1

11. Tallur, B. Analyse des données de l'expression génomique par la classification: pourquoi et comment? in Méthodes et perspectives en classification (2003) Presse académique de Nauchâtel

12. Troyanskaya, O., Cantor, M., Sherlock, G., Brown, P., Hastie, T., Tibshirani,R., Botstein, D., Altman, R. Missing value estimation methods for DNA microarrays Bioinformatics 17 (2001) 520-525 